\title{
Nonarticular Base and Shaft Fractures of Children's Fingers: Are Follow-up X-rays Needed? Retrospective Study of Conservatively Treated Proximal and Middle Phalangeal Fractures
}

\author{
Vonlanthen, Janine ; Weber, Daniel M ; Seiler, Michelle
}

\begin{abstract}
BACKGROUND Phalangeal fractures of the hand are common in children, and most extraarticular fractures can be treated with nonoperative management. Minimally or nondisplaced fractures may simply be immobilized, whereas displaced fractures need closed reduction before immobilization. Although few of these fractures displace secondarily, most schemes currently recommend follow-up x-rays after initial diagnosis. Our primary objective was to identify subgroups of finger fractures that are stable, thus requiring no radiographic monitoring. METHODS This study was designed as a retrospective, single-center analysis of conservatively treated pediatric finger fractures of the proximal and middle phalanges. We included patients up to 16 years with base or shaft fractures of the index to little fingers who underwent nonoperative treatment and standardized follow-up controls in our pediatric hand surgery outpatients' clinic between 2010 and 2016. Fracture angular deformity in x-rays taken at diagnosis and after 1 and 3 weeks were reassessed blinded, and a statistical analysis was conducted to identify fracture types that are prone to secondary angular deformity. RESULTS A total of 478 patients were eligible; 113 were lost due to missing final radiographic controls. Overall, 365 patients were analyzed; they had a mean age of 9.7 years (range, 1 to 16), and $33.4 \%$ required a primary closed reduction. A secondary angular deformity occurred in $2.2 \%(8 / 365)$ of all finger fractures. No secondary angulation occurred in primary minimally and nondisplaced fractures, but $6.6 \%(8 / 122)$ of the reduced fractures showed a subsequent loss of reduction. CONCLUSIONS Minimally angulated ( $<10$ degrees) and nondisplaced metaphyseal and diaphyseal fractures of proximal and middle phalanges of the index to little fingers are stable and therefore do not need radiographic follow-ups. However, initially angulated fractures requiring closed reduction bear a risk of subsequent loss of reduction.
\end{abstract}

DOI: https://doi.org/10.1097/BPO.0000000000001335

Posted at the Zurich Open Repository and Archive, University of Zurich

ZORA URL: https://doi.org/10.5167/uzh-161560

Journal Article

Published Version

Originally published at:

Vonlanthen, Janine; Weber, Daniel M; Seiler, Michelle (2019). Nonarticular Base and Shaft Fractures of Children's Fingers: Are Follow-up X-rays Needed? Retrospective Study of Conservatively Treated Proximal and Middle Phalangeal Fractures. Journal of Pediatric Orthopaedics, 39(9):e657-e660.

DOI: https://doi.org/10.1097/BPO.0000000000001335 


\title{
Nonarticular Base and Shaft Fractures of Children's Fingers: Are Follow-up X-rays Needed? Retrospective Study of Conservatively Treated Proximal and Middle Phalangeal Fractures
}

\author{
Janine Vonlanthen, $M D{ }^{*}+$ Daniel M. Weber, MD, ${ }^{*} \dagger$ and Michelle Seiler, MD广t
}

Background: Phalangeal fractures of the hand are common in children, and most extra-articular fractures can be treated with nonoperative management. Minimally or nondisplaced fractures may simply be immobilized, whereas displaced fractures need closed reduction before immobilization. Although few of these fractures displace secondarily, most schemes currently recommend follow-up x-rays after initial diagnosis. Our primary objective was to identify subgroups of finger fractures that are stable, thus requiring no radiographic monitoring.

Methods: This study was designed as a retrospective, singlecenter analysis of conservatively treated pediatric finger fractures of the proximal and middle phalanges. We included patients up to 16 years with base or shaft fractures of the index to little fingers who underwent nonoperative treatment and standardized follow-up controls in our pediatric hand surgery outpatients' clinic between 2010 and 2016. Fracture angular deformity in $\mathrm{x}$-rays taken at diagnosis and after 1 and 3 weeks were reassessed blinded, and a statistical analysis was conducted to identify fracture types that are prone to secondary angular deformity.

Results: A total of 478 patients were eligible; 113 were lost due to missing final radiographic controls. Overall, 365 patients were analyzed; they had a mean age of 9.7 years (range, 1 to 16), and $33.4 \%$ required a primary closed reduction. A secondary angular deformity occurred in $2.2 \%(8 / 365)$ of all finger fractures. No secondary angulation occurred in primary minimally and nondisplaced fractures, but $6.6 \%(8 / 122)$ of the reduced fractures showed a subsequent loss of reduction.

Conclusions: Minimally angulated ( $<10$ degrees) and nondisplaced metaphyseal and diaphyseal fractures of proximal and middle phalanges of the index to little fingers are stable and therefore do not need radiographic follow-ups. However, initially angulated fractures requiring closed reduction bear a risk of subsequent loss of reduction.

From the *Pediatric Hand Surgery; †Children's Research Center; and $\ddagger$ Pediatric Emergency Department, University Children’s Hospital Zurich, Zurich, Switzerland.

None of the authors received financial support for this study.

The authors declare no conflicts of interest.

Reprints: Michelle Seiler, MD, University Children's Hospital Zurich, Steinwiesstrasse 75, Zurich 8032, Switzerland. E-mail: michelle. seiler@kispi.uzh.ch.

Copyright (C) 2019 Wolters Kluwer Health, Inc. All rights reserved.

DOI: $10.1097 /$ BPO.0000000000001335
Level of Evidence: Level III — retrospective study.

Key Words: finger fractures, phalangeal fractures, follow-up $\mathrm{x}$-rays

(J Pediatr Orthop 2019;00:000-000)

$P$ halangeal hand fractures are the second most common type of fracture during childhood after lower forearm fractures, ${ }^{1-6}$ and finger fractures are the most frequent fracture type in children between 10 and 14 years. When a phalangeal fracture is suspected, a meticulous examination for possible rotational deformity is paramount. This is followed by posteroanterior (PA) and lateral radiographs of the finger. ${ }^{1,4}$

Treatment approaches differ depending on the type of phalangeal fractures. Operative treatment is generally necessary in both intra-articular and unstable fractures, but nonoperative management is the treatment of choice in the vast majority of extra-articular phalangeal fractures. ${ }^{5,7-9}$ Minimally and nondisplaced fractures can be immobilized and treated conservatively. Fractures with greater initial displacement can be treated similarly, but they require closed reduction before immobilization.

Current recommendations for pediatric patients are sparse concerning follow-up radiographs of base and shaft fractures of the proximal and middle phalanges. The standard follow-up regimens include clinical and radiographic monitoring after 1 and 3 weeks. ${ }^{9-11}$ However, our experience has shown that most conservatively treated phalangeal fractures heal without secondary displacement. Therefore, the aim of this study was to assess which subgroup of phalangeal fractures are stable and thus do not need any follow-up radiographs.

\section{METHODS}

\section{Study Design}

This study was designed as a retrospective, singlecenter analysis of conservatively treated pediatric finger fractures between 2010 and 2016. The study was approved by the local ethics committee. 


\section{Patients}

Our study includes data from children up to 16 years of age with a proximal or middle phalangeal fracture of the index to little finger. Extra-articular, physeal, singular phalangeal fractures were eligible for inclusion in the study. Another inclusion criterion was a parental general consent signed at the initial emergency department (ED) presentation that allowed data to be used for research thereafter. Exclusion criteria were open, multifragmented, articular, and phalangeal neck fractures and multiple phalangeal fractures in one hand. Thumb fractures were excluded because they are less common than fractures of the fingers and the most common thumb fracture in children is a Salter Harris 2 fracture of the metacarpal with an inherent tendency to displacement.

A standard protocol was followed for the treatment of finger fractures. Any primary displaced fracture with an angle of $>25$ degrees in the dorsopalmar plane, $>15$ degrees in the radioulnar plane, or a malrotation required closed reduction before immobilization. ${ }^{10,11}$ This initial treatment was managed by experienced ED physicians. Rotational deformity was assessed clinically by observing the planes of the nail beds and digital overlap during active finger flexion. All fractures were immobilized either with a forearm-based volar hand splint in an intrinsic plus position or with buddy taping for 3 weeks. The decision to use taping or splinting for immobilization was made at random as part of a prospective study between 2011 and 2016 regarding the outcome of taping versus splint immobilization. ${ }^{12}$ The finger fractures were monitored by clinical examinations and radiographs after 1 and 3 weeks at the pediatric hand surgery outpatients' clinic. Consequently, a set of $x$-rays included radiographs at initial presentation, postreduction, day $7( \pm 2 \mathrm{~d})$, and day $23( \pm 2 \mathrm{~d}){ }^{9-11}$

We first determined the variables to be analyzed for each patient. These variables were the type of fracture (shaft, metaphysis), phalanges (proximal, middle), angulation (dorsopalmar planes, radioulnar planes, and rotation), degree of angular deformity, reduction necessity, type of immobilization, and occurrence of secondary angular deformity (in days). All variables were gathered from the reassessment of each x-ray except for the type of immobilization and the occurrence of a rotational deformity, which were found in the electronic patient chart. The data were anonymized and transcribed into SPSS.

\section{Statistical Analyses}

All x-rays were reassessed with a standard measuring technique to ensure precision and accuracy. One line was drawn parallel to the epiphyseal plate and another parallel to the phalangeal shaft. The angle between these lines was measured; its deviation from 90 degrees is the fracture's initial angular deformity (Fig. 1). This procedure was performed with both PA and lateral views on x-rays.

A measurement-consistency analysis was conducted in a preliminary series of 50 patients with $3 \mathrm{x}$-rays each to ensure consistent angle measurement between the 5 reviewers ( 2 doctors and 3 study nurses). The maximum acceptable difference between measurements was set in

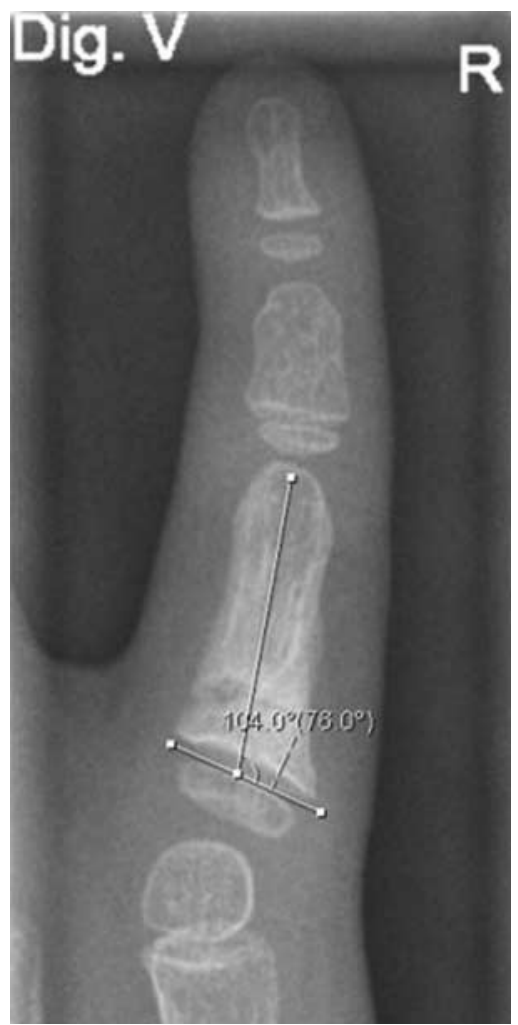

FIGURE 1. Depiction of fracture angulation measuring technique. Parallel lines are drawn through the epiphyseal plate and the phalangeal shaft. The deviation from a 90-degree angle between these 2 is the fracture angulation. This example shows a fracture angulation of 14 degrees. The x-rays were assessed electronically using a picture archiving and communication system (PACS).

advance at 3 degrees. A Bland-Altman analysis was performed on the differences between measured angles. ${ }^{13}$ This indicated that the measurement differences between the reviewers lay within the predefined maximum of 3 degrees.

We then measured the angulation in all $x$-rays. At least $3 \mathrm{x}$-rays series were available for each patient. To eliminate any observer bias that could occur when consecutively measuring a single patient's follow-up x-rays, each x-ray was measured by a different reviewer.

Values were missing from about $25 \%$ of the measurements in our data, which was due either to a missing second consultation or to inapplicable measurements due to rotated $\mathrm{x}$-rays. Therefore, multiple imputation was used to create a more regular data set to aid model-fitting. To verify the stability of the models, imputation was performed twice for the exploratory analysis using the mice package in R. ${ }^{14}$ Multiple imputation approach was chosen because it is superior to the list-wise deletion approach. This is because results based on multiple imputation are valid under both missing completely at random and also the much weaker missing at random assumption on the absence of data. In contrast, list-wise deletion is only valid under the stronger missing completely at random assumption. ${ }^{14}$ 
To model the occurrence of secondary angular deformity, our logistic regression used the angles of dorsopalmar and radioulnar planes and the binary indicator rotational deformity as predictor variables. A 10-fold imputation was performed, and the pooled $P$-values were analyzed.

The statistical analysis was performed by a statistical team from the ETH (StatsLab, ETH).

\section{RESULTS}

From a total of 478 patients, 113 were lost due to the absence of final follow-up; the remaining 365 patients had a mean age of 9.7 years (range, 1 to $16 \mathrm{y}$ ).

Overall, the little finger was fractured most frequently $(74.8 \% ; 273$ cases), followed by the ring and index fingers $(9.3 \% ; 34$ cases each), and the middle finger $(6.6 \%$; 24 cases). In $83 \%$ of all cases, these phalangeal fractures occurred in the proximal phalanx; the middle phalanx was affected in the remaining $17 \%$. The base of the phalanx was injured in $88.8 \%$ of all cases, and the remaining $11.2 \%$ involved fractures in the shaft. The fracture pattern included $82.7 \%$ Salter Harris 2, $8.5 \%$ oblique, 2.7\% transverse, $2.7 \%$ buckle fractures, $2.2 \%$ fractures just distal to the physis, and $1.1 \%$ metadiaphyseal fractures.

A closed reduction of primary angulated fractures was necessary in $33.4 \%$ (122 patients), this procedure was performed under nitrous oxide sedation by ED physicians. In total, $63 \%$ were reduced for dorsopalmar angulation, $23 \%$ for radioulnar angulation, and $14 \%$ for malrotation. Of these reduced finger fractures, only $6.6 \%$ (8 patients) had a subsequent loss of reduction, and these were detected during the first clinical and radiographic controls. All these cases required internal fixation with Kirschner wires. None of the minimally or nonangulated fractures showed a secondary angular deformity.

All patients with subsequent loss of reduction presented with an initial angular deformity of $>10$ degrees, mostly in addition to a malrotation $(87.5 \%)$. Although the logistic regression did not show a statistically significant association between the initial angulation and the occurrence of secondary angular deformity, we were still able to show a correlation between these factors, as seen in Figure 2. An initial angulation $>10$ degrees presents a greater risk of secondary angular deformity than one of 10 degrees or less.

\section{DISCUSSION}

All minimally and nondisplaced finger fractures of the metaphysis and diaphysis of the index to little fingers in our sample were stable and healed without secondary angular deformity. These results indicate that follow-up radiographs are likely to be redundant for these fracture types.

One third of the phalangeal fractures in our study initially required closed reduction. Even without osteosynthesis, this procedure has been shown to produce good outcome in children, as the periosteum in pediatric patients provides support for stabilization, so no internal fracture fixation is necessary. $7,9,10$ These assertions are

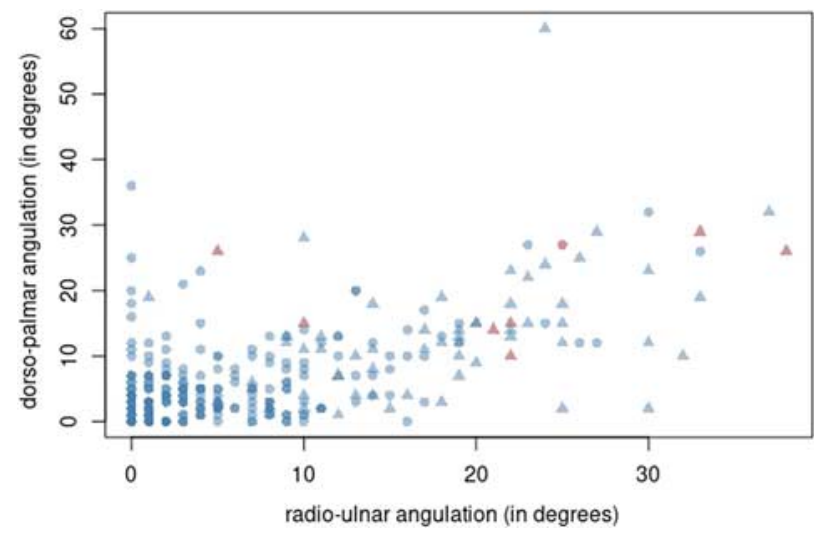

FIGURE 2. Plot diagram of initial angular deformity and secondary angulation. The dorsopalmar and radioulnar angulations of initial x-rays are plotted along the 2 axes. Darker plots represent a higher density of plots. Rotational deformity is shape-coded with a triangle, and the occurrence of secondary angulation color-coded, showing no secondary angulation below an angular deformity of $<10$ degrees. $\frac{\text { full color }}{0 \mathrm{nline}}$

validated by the results of our study: Secondary angular deformity occurred in only 8 patients, all of whom initially required closed reduction $(6.6 \%, 8 / 122)$. Because of the small number of secondary angular deformities we are unable to confirm the correlation of primary fracture angulation with the occurrence of secondary angular deformity at a $95 \%$ confidence interval.

Previous studies have suggested treating minimally displaced and nondisplaced finger fractures of the base and shaft of the proximal and middle phalanges with a 3-week immobilization and radiographic controls. $8,10,11,15$ The identification of a stable subgroup of pediatric finger fractures enables new follow-up regimens for these fracture types to be developed. However, the definition of thresholds is challenging for follow-up radiographs based on primary angular deformity. This difficulty is due to the varying severity of angulation in the dorsopalmar and radioulnar planes; a fracture angular deformity has greater potential for correction in the dorsopalmar plane than in the radioulnar plane. ${ }^{16}$ As Figure 2 illustrates, all 8 patients with secondary fracture angular deformities initially showed a severely angulated fracture, and 7 of 8 exhibited an additional rotational deformity. Hence, a meticulous examination for possible rotational deformity is paramount, as it seems to be strongly associated with secondary angular deformity. Interestingly, the secondary angular deformity in all 8 patients was detected during the first clinical and radiographic controls. Therefore, the question arises whether a single radiographic follow-up would also be sufficient for primarily reduced finger fractures. Remodeling in the radioulnar plane is very limited, even in children and there are no data regarding the degree of secondary displacement after reduction of fractures. Therefore, the $\mathrm{x}$-ray 1 week after reduction should not be skipped even if some of the displaced fractures would actually remodel adequately. Further studies would be necessary to support an informed assertion. 
The radiation exposure of the majority of children with finger fractures would be reduced by omitting followup radiographs of minimally or nondisplaced finger fractures. In fact, a child with a minimally or nondisplaced finger fracture would only undergo $2 \mathrm{x}$-rays (PA and lateral views) at initial ED presentation instead of up to 6 as currently. Furthermore, general medical costs and consultation time would be reduced. Thus far, our standard of monitoring finger fractures has been to see every patient for a clinical and radiographic consultation at our outpatients' clinic. In future, children with minimally or nondisplaced finger fractures will forgo radiographic follow-ups and receive only a clinical consultation with the family's pediatrician after completing a 3-week immobilization.

The major limitations of this study are its retrospective design and the small number of secondary angular deformities, which limits the interpretation and generalization of results. Furthermore, 113 patients were lost to follow-up.

\section{CONCLUSIONS}

Minimally angulated $(<10$ degrees $)$ and nondisplaced metaphyseal and diaphyseal fractures of proximal and middle phalanges of the index to little fingers are stable, and they need no radiographic follow-up controls. In contrast, initially angulated fractures that require closed reduction before immobilization should be checked by radiographic follow-ups as they bear the risk of subsequent loss of reduction.

\section{ACKNOWLEDGMENTS}

The authors thank Dominique Anderegg, Sévérine Bader, and Sylvia Breitenmoser for their assistance in data collection. The authors thank Markus Kalisch and his students Adeline Fermanian, Chris Salahub, and Kay Spiess for the statistical analysis.

\section{REFERENCES}

1. Abzug JM, Dua K, Bauer AD, et al. Pediatric phalanx fractures. J Am Acad Orthop Surg. 2016;24:e174-e183.

2. Verver D, Timmermans L, Klaassen RA, et al. Treatment of extra-articular proximal and middle phalangeal fractures of the hand: a systematic review. Strategies Trauma Limb Reconstr. 2017;12:63-76.

3. Singh J, Jain K, Ravishankar R. Outcome of closed proximal phalangeal fractures of the hand. Indian J Orthop. 2011;45:432-438.

4. Gaston RC, Chadderton C. Phalangeal fractures displaced/nondisplaced. Hand Clin. 2012;28:395-401.

5. Carpenter S, Rohde RS. Treatment of phalangeal fractures. Hand Clin. 2013;29:519-534.

6. Naranje SM, Erali RA, Warner WC Jr, et al. Epidemiology of pediatric fractures presenting to emergency departments in the United States. J Pediatr Orthop. 2016;36:e45-e48.

7. Cornwall R, Riccetti ET. Pediatric phalanx fractures-unique challenges and pitfalls. Clin Orthop Relat Res. 2006;445:146-156.

8. Mahabir RC, Kazemi AR, Cannon WG, et al. Pediatric hand fractures: a review. Ped Emerg Care. 2001;17:153-156.

9. Boyer JS, London DA, Stepan JG, et al. Pediatric proximal phalanx fractures: outcomes and complications after the surgical treatment of displaced fractures. J Pediatr Orthop. 2015;35:219-223.

10. Oetgen ME, Dodds SD. Non-operative treatment of common finger injuries. Curr Rev Musculoskelet Med. 2008;1:97-102.

11. Kozan SH, Waters PM. Fractures and dislocations of the hand and carpus in children. In: Beaty JH, Kasser JR, eds. Rockwood and Wilkins' Fractures in Children. Philadelphia, PA: Lippincott Williams \& Wilkins'; 2010:225-255.

12. Weber DM, Seiler M, Subotic U, et al. Buddy taping versus splint immobilization for pediatric finger fractures: a randomized controlled trial. J Hand Surg Eur Vol. 2018. [In press].

13. Bland JM, Altman DG. Statistical methods for assessing agreement between two methods of clinical measurement. Lancet. 1986;1:307-310.

14. van Buuren S, Groothuis-Oudshoorn K. MICE: multivariate imputation by chained equations in R. J Stat Soft. 2011;45:1-67.

15. Lindley SG, Rulewicz G. Hand fractures and dislocations in the developing skeleton. Hand Clin. 2006;22:253-268.

16. Laer LK, Kraus R, Linhart WE. Frakturen und Luxationen im Wachstumsalter. Stuttgard, PA: Thieme; 2012. 